\title{
Passive Frequency Selective Surface Array as a Diffuser for Destroying Millimeter Wave Coherence
}

\author{
Saiful Islam, Johan Stiens, Gert Poesen, Irina Jaeger, and Roger Vounckx \\ Laboratory for Micro- and Photonelectronics, Department of Electronics and Informatics, Vrije Universiteit Brussel, Pleinlaan 2, \\ 1050 Elsene, Belgium
}

Correspondence should be addressed to Saiful Islam, sislam@etro.vub.ac.be

Received 30 November 2007; Accepted 15 May 2008

Recommended by Tibor Berceli

This paper presents the design, construction, and testing of grounded frequency selective surface (FSS) array as a diffuser for destroying millimeter wave coherence which is used to eliminate speckle in active millimeter wave imaging. To create stochastically independent illumination patterns, we proposed a diffuser based on random-phase distributions obtained by changing the incident frequency. The random-phase diffuser was obtained by mixing up the phase relations between the cells of a deterministic function (e.g., beam splitter). The slot length of FSS is the main design parameter used to optimize the phase shifting properties of the array. The critical parameters of the diffuser array design, such as phase relation with slot lengths, losses, and bandwidth, are discussed. We designed the FSS arrays with finite integral technique (FIT), fabricated by etching technique, and characterized the $S$-parameters with a free-space MVNA, and measured the radiation patterns with a BWO in motorized setup.

Copyright (C) 2008 Saiful Islam et al. This is an open access article distributed under the Creative Commons Attribution License, which permits unrestricted use, distribution, and reproduction in any medium, provided the original work is properly cited.

\section{INTRODUCTION}

Free-space active millimeter wave (mm-wave) systems have gained more and more attraction during the last few years due to their indoor security applications. There is no incoherent mm-wave source, and highly coherent $\mathrm{mm}$-wave sources produce speckle in active mm-wave imaging of conceal objects because of interference phenomenon [1]. The speckle problem is especially important for active $\mathrm{mm}$ wave imaging as the wavelength in this frequency range is close to the object dimension [2, 3]. We present here a frequency selective surface (FSS)-based diffuser array to destroy the coherence of $\mathrm{mm}$-wave sources. This optical technique utilizes stochastically independent phase patterns obtained from a grounded FSS array. Nongrounded FSSs have limited bandwidth and can be viewed as filters for plane waves at any angles of incidence [4]. They are well known in the literature for their filtering characteristics at microwave and millimeter wave frequencies. Generally, FSSs are based on two-dimensional periodic lattice decorated with resonant elements-including dielectric or metallic circuit designs [5]. This paper considers the building block of a simpler and more versatile architecture, where the reflection phase depends on the resonant slot length. Such elements are widely employed as grounded FSS. Using ground plane to the back side of FSS and also by choosing the proper slot dimensions and unit cell dimensions, such structure can be designed for full W-band $(75-110 \mathrm{GHz})$ application. The resonant frequency and the phase of such slotted FSSs can be controlled by varying slot lengths $[6,7]$. The phase variation with slot length variation is more significant near the resonant frequency [7]. This property of the grounded FSS is important to design a random-phase diffuser to destroy the coherence of mm-wave sources. So, it is a novel idea to use FSS cells with different slot length to control the phase of individual element of a diffuser array. A diffuser system is an antenna array which receives coherent plane wave and reflects noncoherent wave by introduction different phase delay in the reflected wave at each single frequency within the W-band. The diffuser applies the temporal phase variation to the reflected wave. Actually, due to the different amount of phase delay, the coherent plane wave after reflection will convert to noncoherent illumination. The diffuser is like a phase shifter to modulate the phase properties of coherent wave. If we excite the proposed array with a monochromatic wave (single-frequency), the reflected 


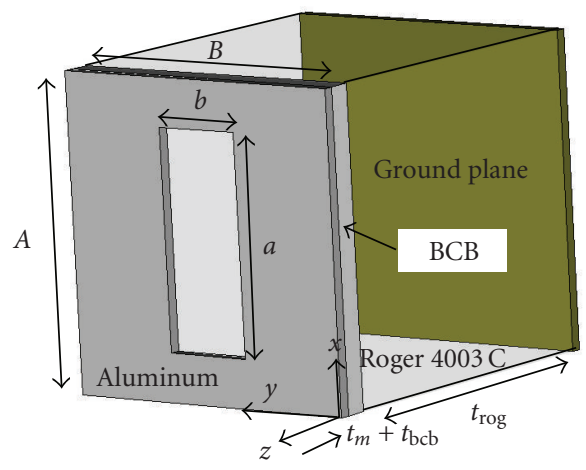

Figure 1: Unit cell of rectangular slot grounded FSS.

wave is still a monochromatic, but the phase pattern will be different, and the amplitude will be direction-dependent. To design such a diffuser different phase delay, elements are needed. In the proposed diffuser system, the delay element is replaced with FSS cells of different slot lengths.

Reflect array with patch antenna requires tight fabrication tolerances to achieve desired phase value, as the patch size versus phase curves are extremely nonlinear [8]. Because of the rapid phase change around resonance, most reflectarray elements have lengths within $\pm 5 \%$ of the nominal resonant length $[8,9]$. This causes phase errors which results for changes in frequency greater than a few percent. Random phase errors due to etching tolerances, is usually more critical for patch antenna than Slot FSS because of the large slope of the phase versus patch-size curves. This effect also limits the bandwidth. So, it is difficult to design deterministic function or to get controlled phase response at the desired frequency. By using thick substrate, the slope of phase versus patch size curve can be reduced but the quality factor $(Q)$ and the total phase range decrease. As explained in [10], the dual resonant response of a two layergrounded FSS array of dissimilar size patch elements was used to overcome the limitations associated with the use of thick substrates, but the structure cannot be printed on a single substrate surface; double-side mask alignment is needed. This means difficulties with the design accurately. The present design excludes the possibility of obtaining dual frequency operation since independent control of the dimensions of the slot length is normally required in each unit cell. It is easier than multilayer design in "[10]." The phase versus frequency curve of grounded FSS is more linear so no need of tight fabrication tolerances like patch antenna. The proposed periodic array structure can be printed on a single substrate surface; double-side mask alignment is not needed [11]. The structure is the superior alternative to other broadband elements as the construction of the structure is very much simpler and cheaper.

This paper is organized as follows. Section 2 describes the design, fabrication, and testing of slot length dependence phase variation of grounded FSS. In practice, the designs of FSSs with different slot lengths show that FSS can be used as phase-delay circuit. In Section 3, the same concept is applied to design an FSS array compose of different slot lengths to split the coherent mm-wave beam for a deterministic function analysis. In Section 4, the FSS cells of the beam splitter array explained in Section 3 were rearranged to get a stochastically independent phase patterns. The reshuffled array gives diffused radiation pattern at every frequency in $\mathrm{W}$-band which proves that the proposed coherent destroyer is capable to destroy the coherence of $\mathrm{mm}$-wave by generating random-phase reflection.

\section{W-BAND GROUNDED FSSs}

The design of W-band quasioptical filters, consisting of periodically perforated slots on metal backed Roger 4003C substrate, is considered. As shown in Figure 1, the structure consists of rectangular slots in $1.5 \mu \mathrm{m}$, aluminum on top of $1489 \mu \mathrm{m}$ grounded Roger 4003C substrate of dielectric constant $\varepsilon_{r}=3.38$, and loss tangent 0.0027 . Before metallization, a $10 \mu \mathrm{m}$ benzo-cyclo-butene (BCB) layer was deposited between the aluminum layer and Roger 4003C to level the roughness of the Roger 4003C surface. As the thickness of the dielectric wafer Roger 4003C is in the order of the half wavelength, the structure behaves like a FabryPerot resonator. During the simulation, the structure can be tuned out so that the FSS geometry can be adapted to a slightly different wafer thickness, without deterioration of the device characteristics. The resonant frequency can be set properly by choosing the space between the slots ( $A$ and $B$ in Figure 1) and the slot length " $a$ " and slot width " $b$." The simulations of the structure were carried out by using commercial CST microwave studio software. Starting the simulation with initial values obtained from calculation of the approximate resonance condition, final values were determined from CST simulation results. The unit cell dimensions were optimized to the value of $A=B$ $=1400 \mu \mathrm{m}$.

We considered three grounded FSSs with slot lengths of $896 \mu \mathrm{m}, 970 \mu \mathrm{m}$ and $1076 \mu \mathrm{m}$ and their sloth width of $400 \mu \mathrm{m}$ to check the slot length dependence phase variation. These values are obtained from the CST simulation. The CST simulation software performs simulation by using numerical tools of finite integral technique (FIT). In this case, the numerical method provides a universal spatial discrimination scheme, applicable to various electromagnetic problems, ranging from static field calculations to highfrequency applications in time domain. FIT is static up to the $\mathrm{THz}$ range. Due to the fact that a computer is only capable of calculating problems which have finite expansion, we need to specify the boundary conditions. The basic functions are calculated numerically by using the perfect boundary approximation. The perfect boundary conditions were chosen to get the tangential electric and tangential magnetic fields components uniform inside the slot but outside to zero. The electric boundary $E_{t}=0$ (i.e., all tangential electric fields and normal magnetic fluxes are set to zero) was chosen in the " $y$ " axis direction and the perfect magnetic boundary $H_{t}=0$ (i.e., all tangential magnetic fields and normal electric fluxes are set to zero) was chosen in the " $x$ " axis direction as in Figure 1. 


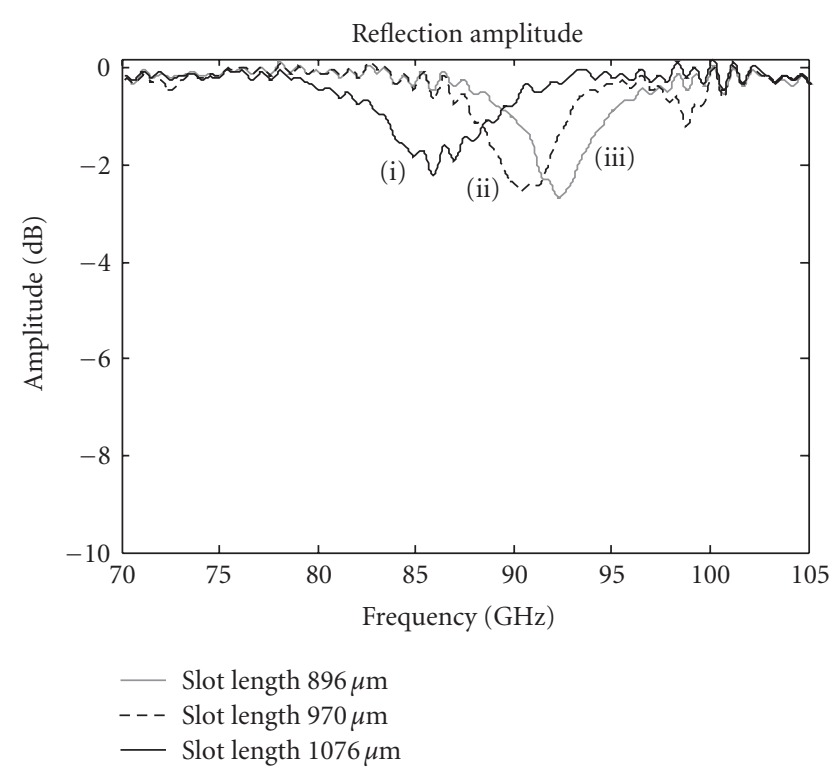

FIgURE 2: Measured reflection amplitudes of different slot length grounded FSSs. (Slot length of curve (i) $1076 \mu \mathrm{m}$, curve (ii) $970 \mu \mathrm{m}$, and curve (iii) $896 \mu \mathrm{m}$.)

\subsection{Measurement results of FSSs}

As discussed in our paper [7], the measurement amplitude and phase of a grounded FSS fit well with simulation results. To show the slot length dependence phase variation (i.e., phase delay), only the measurement amplitude and phase curves of the FSSs measured with free-space millimeter wave vector network analyzer (MVNA) are presented here. The reflection amplitudes of the three FSSs are shown in Figure 2. The minimum reflections $\left(S_{11}\right)$ at resonant frequencies are $-2.17 \mathrm{~dB},-2.53 \mathrm{~dB}$, and $-2.68 \mathrm{~dB}$ for curves (i), (ii), and (iii), respectively, which prove that the FSSs reflect in full $\mathrm{W}$-band, and the total W-band phase is useable in reflection mode. The realization of slot FSS in compare to the patch demonstrate that for FSS, the maximum transmission occurs at the resonant frequency. Therefore, there are losses at resonance since some portion of the energy is lost in the area between FSS and the ground plane. The FSS suffers higher losses at the resonance in compare to patch, but the phase variation with frequency of the FSS is more linear than patch antenna. This property is more advantageous than the higher reflection gain.

Figure 3 shows the effect of slot length variation on phase of FSSs in W-band. Near resonance, a small change of resonant slot length causes a large phase variation in the FSS phase curve. For example, in Figure 3, at $91.61 \mathrm{GHz}$, the phase values of the curves (i), (ii), and (iii) are $-259.3^{\circ}$, $-176.6^{\circ}$, and $-79.16^{\circ}$, respectively. So, the phase delay between the curve (i) and curve (iii) is $180^{\circ}$. At $91.61 \mathrm{GHz}$, the slot length variation of $106 \mu \mathrm{m}$ [curve (i)-(ii)] gives a phase delay 82.7 degree, and resonance slot length difference of $180 \mu \mathrm{m}$ [(i)-(iii)] gives a phase delay of 180 degree. Figure 3 also shows that by changing the slot lengths, the phase delay and phase gradient can be controlled. We

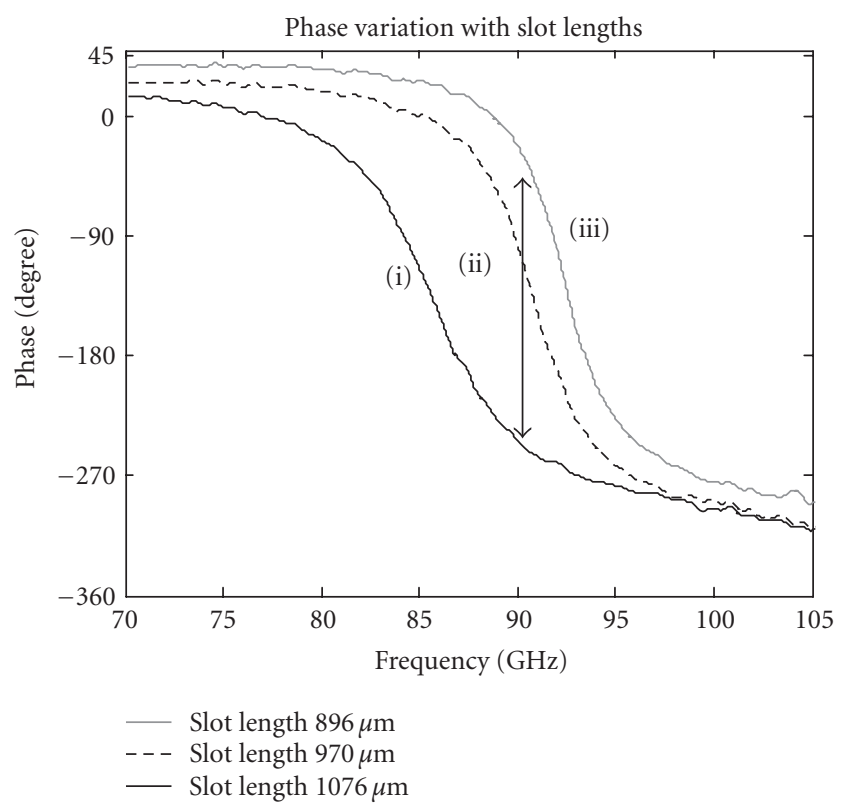

FIgURE 3: Measured phase versus frequency curves, slot lengths as variable parameter. (Slot length of curve (i) $1076 \mu \mathrm{m}$, curve (ii) $970 \mu \mathrm{m}$, and curve (iii) $896 \mu \mathrm{m}$.)

measured total $350^{\circ}$ phase variation in the $\mathrm{W}$-band. This value can be increased by decreasing the slot length and by introducing higher order modes.

\section{DETERMINISTIC FUNCTION}

Till now, we have shown how phase delay can be introduced by using FSS of different slot lengths. This investigation demonstrates that each FSS cell with unique slot length is equivalent to a delay element, and the amount of delay is determined by the slot length. As an example of deterministic function realization, mm-wave beam splitter by FSS array is consider in this section. The idea of this design is to introduce variable phase delay in constant phase of a coherent $\mathrm{mm}$ wave plane wave to split the beam in two directions to observe the phase-delay effect. The schematic diagram of the beam splitter array is shown in Figure 4, where every pattern represents a different slot length. As the slot length variation is in the order of micron and is less significant in figure, schematic diagrams are presented instead of real photo arrays. The beam splitter array was designed with $20 \times 20$ cells of FSS composite of 20 different slot lengths, divided in to two subarrays as shown in Figure 4. Each pattern of Figure 4 represents unique slot length. The column wise patterns represent different slot lengths and their phase values are listed in Table 1 . In the left $10 \times 20$ cells subarray, all the cells have negative phase values and in the right 10 $\times 20$ cells subarray, all cells have positive phase values. The different length FSS cells were distributed column wise in such a way that from the center of the array, the phase delay will increase in both left and right sides of the array shown in Figure 4. Table 1 shows the slot lengths of FSS cells, their corresponding phase values (obtained from CST simulations at $94 \mathrm{GHz}$ ), and the respective phase delays. The 
TABLE 1: Slot lengths, corresponding phase values, and phase delays of beam splitter. The patterns of the left most column of the table represent different columns of Figure 4.

\begin{tabular}{|c|c|c|c|}
\hline & Phase (degree) & Slot length $(\mu \mathrm{m})$ & Delay (degree) \\
\hline$=$ & $-138^{\circ}$ & 1097 & $-61^{\circ}$ \\
\hline II & $-128^{\circ}$ & 1048 & $-54^{\circ}$ \\
\hline $\mathscr{W}$ & $-116^{\circ}$ & 1013 & $-47^{\circ}$ \\
\hline$=$ & $-103^{\circ}$ & 990 & $-40^{\circ}$ \\
\hline III & $-89^{\circ}$ & 972 & $-34^{\circ}$ \\
\hline$N$ & $-75^{\circ}$ & 959 & $-29^{\circ}$ \\
\hline W/ & $-61^{\circ}$ & 949 & $-23^{\circ}$ \\
\hline 册 & $-47^{\circ}$ & 941 & $-17^{\circ}$ \\
\hline$\square$ & $-30^{\circ}$ & 931 & $-11^{\circ}$ \\
\hline & $0^{\circ}$ & 917 & $0^{\circ}$ \\
\hline њ & $32^{\circ}$ & 903 & $32^{\circ}$ \\
\hline & $49^{\circ}$ & 895 & $49^{\circ}$ \\
\hline$\beta$ & $63^{\circ}$ & 888 & $63^{\circ}$ \\
\hline \% & $78^{\circ}$ & 879 & $78^{\circ}$ \\
\hline & $91^{\circ}$ & 870 & $91^{\circ}$ \\
\hline 88 & $105^{\circ}$ & 858 & $105^{\circ}$ \\
\hline 2 & $116^{\circ}$ & 846 & $116^{\circ}$ \\
\hline 88 & $127^{\circ}$ & 832 & $127^{\circ}$ \\
\hline & $138^{\circ}$ & 812 & $138^{\circ}$ \\
\hline 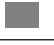 & $147^{\circ}$ & 785 & $147^{\circ}$ \\
\hline
\end{tabular}

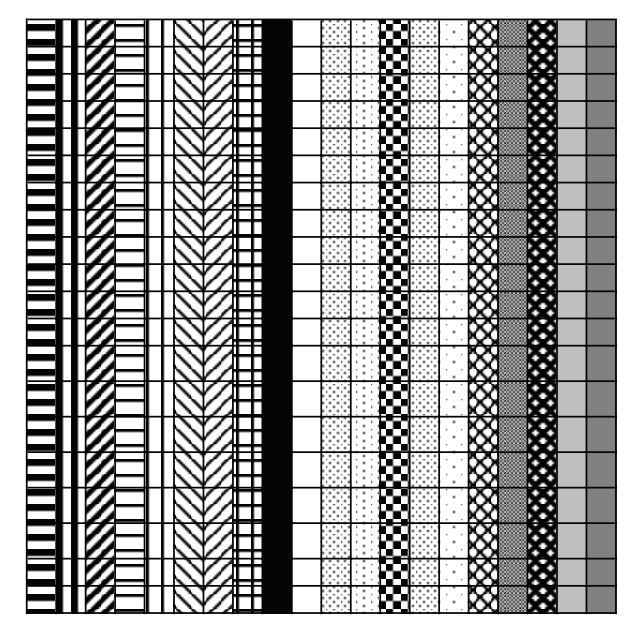

FIGURe 4: Schematic drawing of beam splitter FSS array. (Patterns follow the values listed in Table 1.)

amounts of phase delays were calculated by using the formula of geometrical optics, considering the interspacing distances of the FSS cells (i.e., distance between the centers of two successive unit cells) and for an off axis beam focal distance of $30 \mathrm{~cm}$ from the antenna surface. The FSS array was designed in a way that both subarrays will bend the beam and will focus $30 \mathrm{~cm}$ away from the antenna face. Due to left and right bending, splitted radiation will be obtained in each frequency.

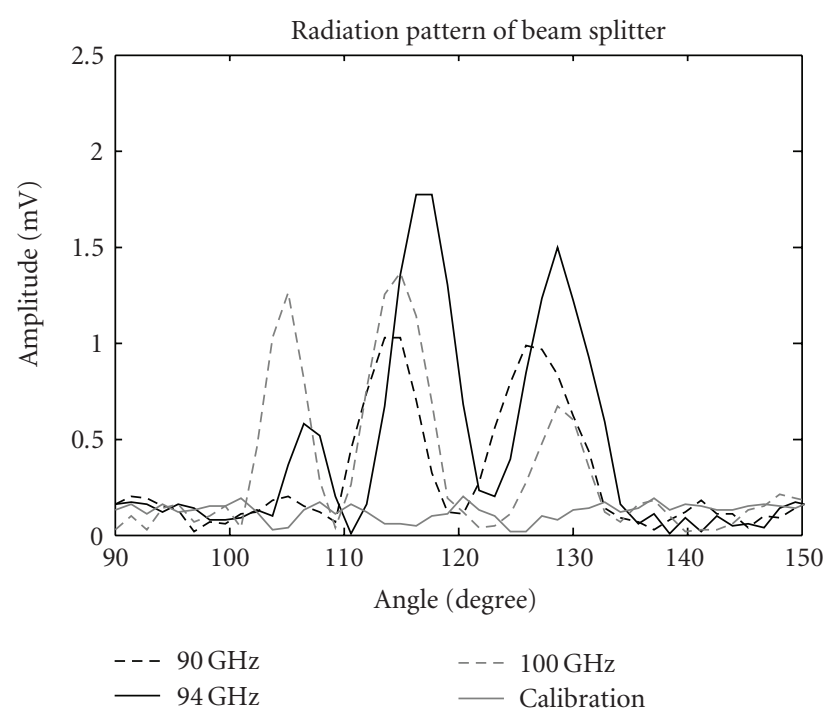

(a)

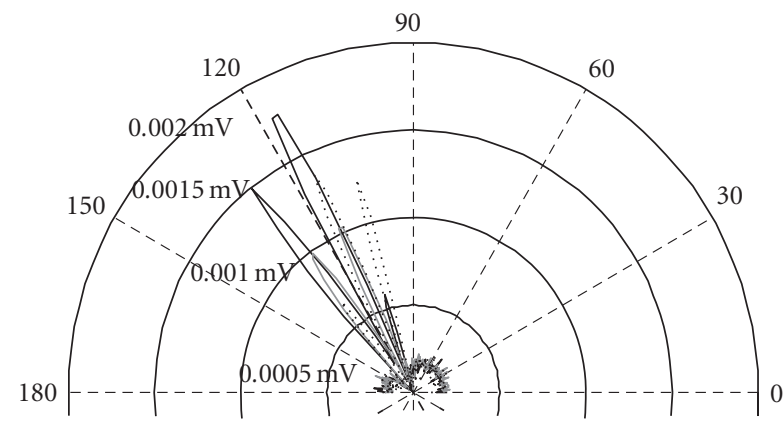

(b)

FIGURE 5: Measured near field radiation pattern of beam splitter array at different incident frequencies: (a) rectangular plot (b) polar plot.

\subsection{Measurement results of beam splitter}

The radiation patterns of the arrays were measured with backward-wave oscillator (BWO) in motorized setup. The measurement was carried out with $45^{\circ}$ angular position of the antenna axis to the BWO millimeter wave source axis (in measurement Figures 5(b) and 7(b)). The antenna broad side axis was at $135^{\circ}$. At $94 \mathrm{GHz}$ measurement, two lobes were obtained at $130^{\circ}$ and $118^{\circ}$ due to the phase variation of beam splitting. The measured rectangular and polar radiation patterns of the beam splitter are shown in Figures 5(a) and 5(b), respectively. In Figure 5(a), the calibration of the measurement system is shown (marked in legend). This is the radiation pattern without antenna and just with the antenna holder. A result shows a good calibration for the antenna measurement setup. 


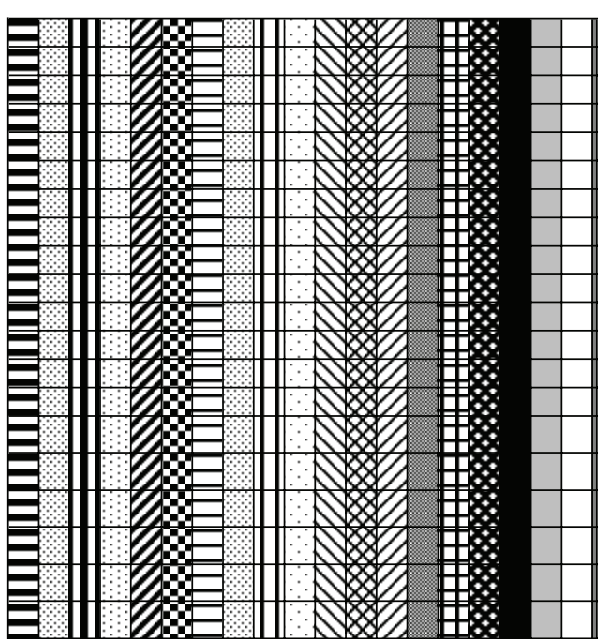

FIGURE 6: Schematic drawing of coherent destroying diffuser. (Patterns correspond to the phase values listed in Table 1.)

The measurement radiation patterns at $90 \mathrm{GHz}, 94 \mathrm{GHz}$, and $100 \mathrm{GHz}$ frequencies are presented. The results show that at each frequency, we get two main lobes on both sides of the antenna broad side axis. The position of lobes changes with frequency as the phase values of the FSS cells changes with frequency. Figure 5(b) shows the radiation patterns in polar plot. Maximum reflection for side lobes is obtained at $94 \mathrm{GHz}$. With the decreasing or increasing of measurement frequency, the side lobes reflection power decreases.

\section{COHERENCE DESTROYING DIFFUSER}

As explained for beam splitter in Section 3, the columnar array of FSS cells mounted on a dielectric substrate, each column represents a delay element. The delay of each column is determined by its slot length. To realize the random phase diffuser system, the columns of beam splitter FSS array of Figure 4 were reshuffled to design the diffuser array. To get random phase pattern, the columns of Figure 4 were the reshuffled as shown in Figure 6.

\subsection{Measurement results of diffuser}

As already mentioned, the amounts of phase delay listed in Table 1 were calculated at $94 \mathrm{GHz}$. So, the change of frequency will introduce different set of phase values at the desired frequency. Due to the random reshuffling of the FSS columns, reflection at each frequency will be diffused reflection, as the delay element of the array introduces random phases unlike focusing of beam splitter. The reflected beams are no longer coherent due to the fact that a random phase introduce from the random positioning of the FSS cells. At each frequency, the reflection is also diffused reflection. The measurement results are shown in Figure 7. The same calibration shown in Figure 5(a) was used for this measurement. For simplicity, the measurement radiation patterns are presented at $85 \mathrm{GHz}, 90 \mathrm{GHz}, 94 \mathrm{GHz}$, $100 \mathrm{GHz}, 105 \mathrm{GHz}$, and $110 \mathrm{GHz}$. Figure 7(a) shows that

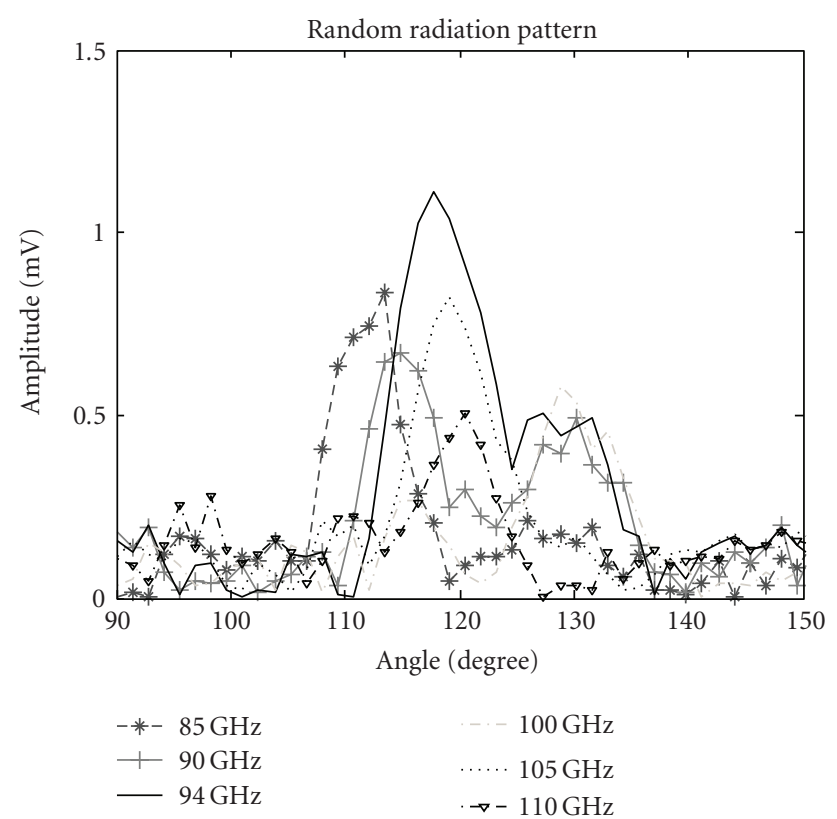

(a)

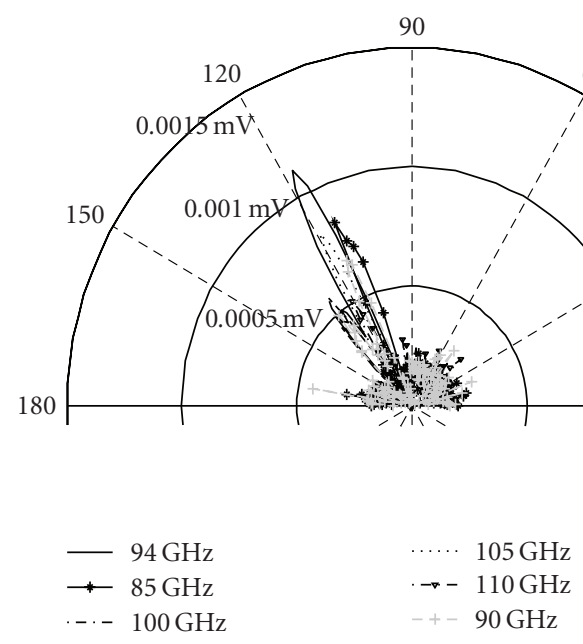

(b)

Figure 7: Measured near field radiation pattern of random phase diffuser: (a) rectangular plot (b) polar plot.

every measurement is different in amplitude direction than the others. The maximum reflection power obtained at $94 \mathrm{GHz}$ and there is no side lobes like the beam splitter. For the sake of representation, the angular position of the detector from $90^{\circ}$ to $150^{\circ}$ is shown in rectangular plots of Figures 5(a) and 7(a).

\section{CONCLUSION}

We have designed a coherence destroying diffuser system using passive FSS array. We explained the slot length dependence phase variation properties of FSS, and also showed how phase delay can be controlled by changing slot lengths. We presented the design of $\mathrm{mm}$-wave beam splitter 
as deterministic function which split the coherent beam in two directions and then shown the coherent destroyer by reshuffling the cell columns of beam splitter array. This coherent destroyer can also be used as multifrequency diffuser system. In multifrequency diffuser system, at each frequency, the radiation pattern is different, and also the reflection is diffused reflection, that is, the reflected signal is incoherent. This type of reflector array is capable to destroy the coherence of the coherent mm-wave sources as the array behaves like a phase modulator which introduces random phases. In multifrequency diffuser approach, the frequency difference should be large enough, since a small difference generates quite similar speckle distributions [12, 13]. The bandwidth of the proposed diffuser is whole W-band, which gives the freedom to use enough frequency differences as multifrequency diffuser.

\section{ACKNOWLEDGMENTS}

This work was partially funded by the Vrije Universiteit Brussel (VUB-OZR), the Flemish Fund for Scientific Research (FWO- G.0041.04), and the Flemish Institute for the encouragement of innovation in science and technology (IWT-SBO 231.011114).

\section{REFERENCES}

[1] I. Ocket, B. Nauwelaers, G. Koers, and J. Stiens, "Fast modeling and optimization of active millimeter wave imaging systems," in Proceedings of the 36th European Microwave Conference (EuMC '06), pp. 1559-1562, Manchester, UK, September 2006.

[2] I. Jaeger, J. Stiens, G. Koers, G. Poesen, and R. Vounckx, "Hadamard speckle reduction for millimeter wave imaging," Microwave and Optical Technology Letters, vol. 48, no. 9, pp. 1722-1725, 2006.

[3] J. I. Trisnadi, "Speckle contrast reduction in laser projection displays," Proc. SPIE, vol. 4657, pp. 131-137, 2002.

[4] S. Islam, J. Stiens, G. Poesen, I. Jäger, and R. Vounckx, "Frequency selective surface as a filter for active millimeter wave imaging," in Proceedings of the 12th Microcol Conference, pp. 137-140, Budapest, Hungary, May 2007.

[5] R. Dubrovka, J. Vazquez, C. Parini, and D. Moore, "Equivalent circuit method for analysis and synthesis of frequency selective surfaces," IEE Proceedings: Microwaves, Antennas and Propagation, vol. 153, no. 3, pp. 213-220, 2006.

[6] S. Biber, M. Bozzi, O. Gunther, L. Perregrini, and L.-P. Schmidt, "Design and testing of frequency-selective surfaces on silicon substrates for submillimeter-wave applications," IEEE Transactions on Antennas and Propagation, vol. 54, no. 9, pp. 2638-2645, 2006.

[7] S. Islam, J. Stiens, G. Poesen, I. Jaeger, G. Koers, and R. Vounckx, "Grounded frequency selective surfaces as W-band quasi-optical filters for active millimeter wave imaging," in Proceedings of the Asia Pacific Microwave Conference, vol. 3, pp. 2401-2404, Bangkok, Thailand, December 2007.

[8] D. M. Pozar and T. A. Metzler, "Analysis of a reflect array antenna using microstrip patches of variable size," Electronics Letters, vol. 29, no. 8, pp. 657-658, 1993.

[9] D. M. Pozar, S. D. Targonski, and H. D. Syrigos, "Design of millimeter wave micro-strip reflect-arrays," IEEE Transactions on Antennas and Propagation, vol. 45, no. 2, pp. 287-296, 1997.
[10] J. A. Encinar, "Design of two-layer printed reflect arrays using patches of variable size," IEEE Transactions on Antennas and Propagation, vol. 49, no. 10, pp. 1403-1410, 2001.

[11] N. Misran, R. Cahill, and V. F. Fusco, "Design optimisation of ring elements for broadband reflectarray antennas," IEE Proceedings: Microwaves, Antennas and Propagation, vol. 150, no. 6, pp. 440-444, 2003.

[12] J. I. Trisnadi, "Hadamard speckle contrast reduction," Optics Letters, vol. 29, no. 1, pp. 11-13, 2004.

[13] E. N. Grossman, A. Luukanen, and A. J. Miller, "Terahertz active direct detection imagers," in Terahertz for Military and Security Applications II, vol. 5411 of Proceedings of SPIE, pp. 68-77, Orlando, Fla, USA, April 2004. 

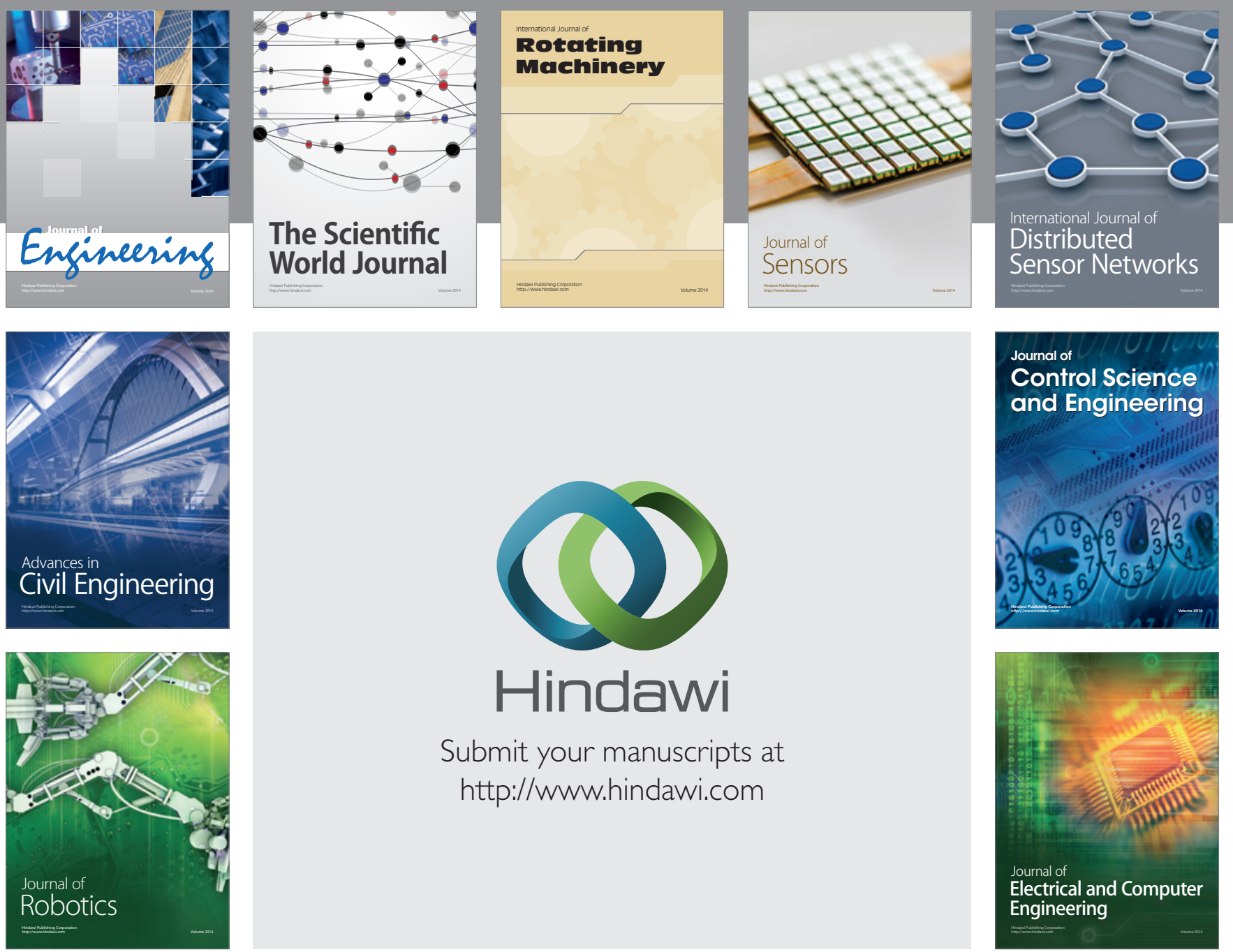

Submit your manuscripts at

http://www.hindawi.com
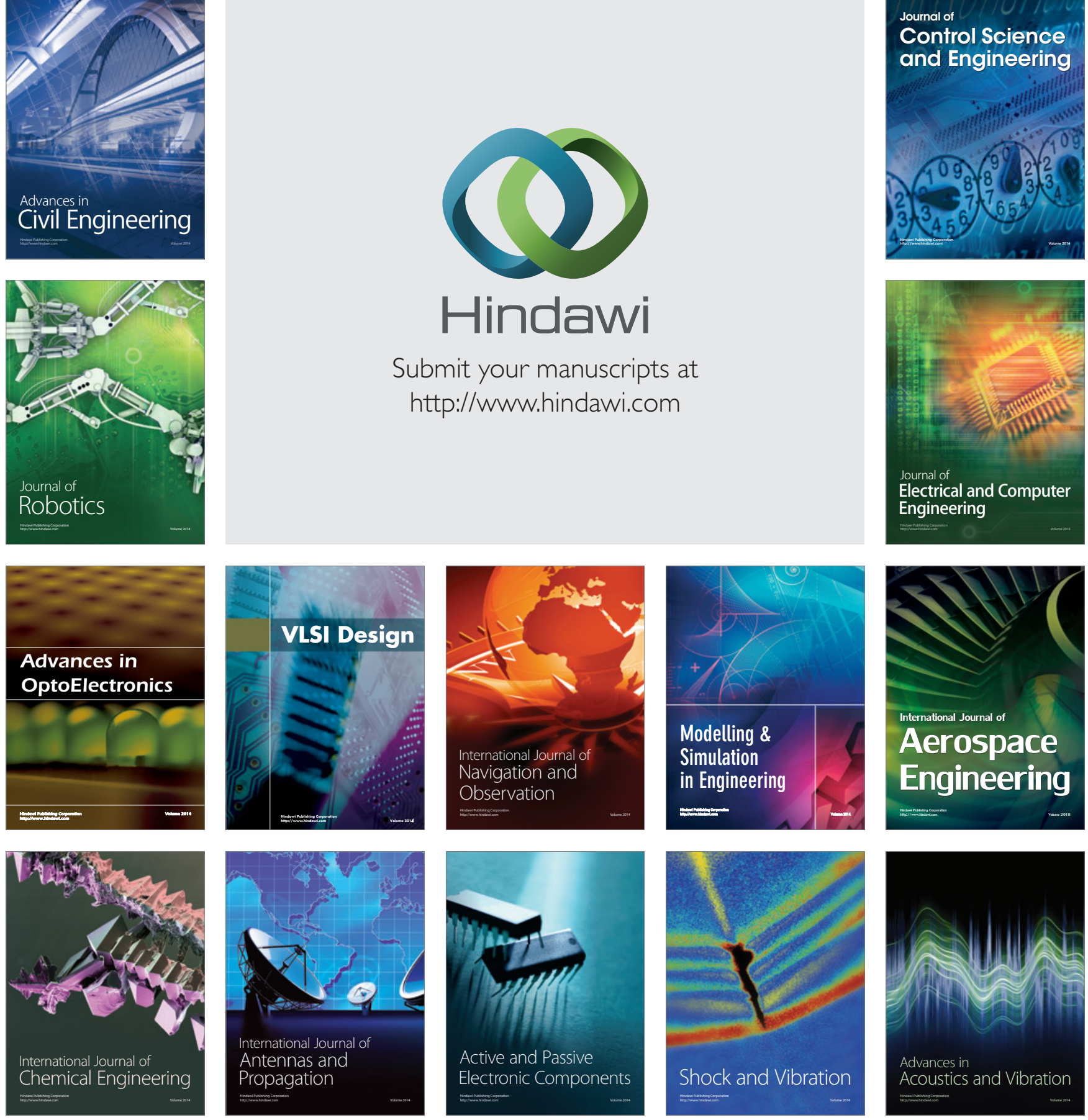